\title{
Comparative mortality risks of antipsychotic medications in community-dwelling older adults ${ }^{\star \dagger}$
}

T. Gerhard, K. Huybrechts, M. Olfson, S. Schneeweiss, W. V. Bobo, P. M. Doraiswamy,

D. P. Devanand, J. A. Lucas, C. Huang, E. S. Malka, R. Levin and S. Crystal

\section{Background}

All antipsychotic medications carry warnings of increased mortality for older adults, but little is known about comparative mortality risks between individual agents.

\section{Aims \\ To estimate the comparative mortality risks of commonly prescribed antipsychotic agents in older people living in the community.}

\section{Method}

A retrospective, claims-based cohort study was conducted of people over 65 years old living in the community who had been newly prescribed risperidone, olanzapine, quetiapine, haloperidol, aripiprazole or ziprasidone $(n=136393)$. Propensity score-adjusted Cox proportional hazards models assessed the 180-day mortality risk of each antipsychotic compared with risperidone.

\section{Results}

Risperidone, olanzapine and haloperidol showed a dose-response relation in mortality risk. After controlling for propensity score and dose, mortality risk was found to be increased for haloperidol (hazard ratio $(H R)=1.18,95 \% \mathrm{Cl}$ 1.06-1.33) and decreased for quetiapine $(\mathrm{HR}=0.81,95 \% \mathrm{Cl}$ 0.73-0.89) and olanzapine ( $\mathrm{HR}=0.82,95 \% \mathrm{Cl} 0.74-0.90)$.

\section{Conclusions}

Significant variation in mortality risk across commonly prescribed antipsychotics suggests that antipsychotic selection and dosing may affect survival of older people living in the community.

\section{Declaration of interest}

P.M.D. has received research grants and advisory/speaking fees from several pharmaceutical companies, including antipsychotic manufacturers, for other research. He owns stock in AdverseEvents Inc. which was not involved in this project. D.P.D. has received research support from Novartis AG and Eli Lilly and has served as a consultant to Bristol-Myers Squibb.
Despite significant safety concerns, ${ }^{1,2}$ and modest evidence of efficacy, ${ }^{3-6}$ antipsychotic medications are widely prescribed off-label in older adults, often for disruptive behavioural symptoms of dementia. ${ }^{7,8}$ In 2005 a meta-analysis of 15 clinical trials in patients with Alzheimer's disease or dementia reported a more than $50 \%$ increase in mortality for second-generation antipsychotics above placebo. ${ }^{1}$ Subsequent observational studies reported equal or greater risk for first-generation agents. ${ }^{2,9-13}$ Starting in 2005, regulatory agencies in the UK and the USA issued warnings of increased mortality risk for off-label use of first- and second-generation antipsychotics in elderly patients with dementia, ${ }^{14,15}$ but use in older adults, including those with dementia, has remained substantial. ${ }^{8,16-19}$ Although the use of antipsychotics is particularly prevalent in nursing homes, more than $70 \%$ of the over 1 million older adults who receive antipsychotics in the USA are treated outside of institutional settings. ${ }^{20,21}$ In England and Wales, approximately $48 \%$ of the more than 100000 older adults without severe mental illness treated with antipsychotics reside in the community, and within one primary care trust $15.3 \%$ of out-patients in a dementia register were recently found to be receiving antipsychotic medications. ${ }^{8,22}$

Much uncertainty remains concerning the comparative safety of individual antipsychotic agents in older adults. Meta-analyses of controlled trials and most observational studies are not adequately powered to compare individual agents with respect to risk of premature mortality. ${ }^{1,2,9-13}$ Using a similar analytic approach to that used in this study, our group recently examined

*Parts of this paper were presented on 17 August 2011 at the 27th International Conference of the International Society for Pharmacoepidemiology in Chicago, Illinois, USA.

†See editorial, pp. 4-5, this issue. comparative mortality risks of antipsychotic medications in nursing-home residents, a frail population with limited life expectancy. ${ }^{23}$ In addition, two prior studies directly or indirectly compared mortality risks of individual antipsychotic agents in predominantly male and community-dwelling older US veterans with dementia. ${ }^{24,25}$ Despite pronounced differences in demographic composition, pattern of comorbidity, cognitive status and frailty between the study populations, these studies reported similar findings regarding the comparative mortality risk of individual medications. In comparison with risperidone, an increased risk was found with haloperidol and a decreased risk with quetiapine, with no significant difference in risk for olanzapine. Dosage is a potentially important determinant of comparative antipsychotic safety. Clinical variations among agents in community dosing patterns may interact with dose response to influence mortality risk. ${ }^{23,24}$ We therefore estimated the comparative non-cancer mortality of the six most commonly prescribed antipsychotic drugs, with and without statistical adjustment for dose, in a national cohort of non-institutionalised older adults enrolled in the US Medicaid and Medicare programmes.

\section{Method}

To compare non-cancer and cause-specific mortality risks between individual antipsychotic medications, we conducted a retrospective, observational cohort study among people 65 years old or older who were living in the community and had recently begun antipsychotic treatment. People with clinical diagnoses of schizophrenia, bipolar disorder or cancer during the 180-day period preceding the index date were excluded. Our study used combined service (Medicare Parts A and B) and pharmacy 
(Medicaid) claims from 1 January 2001 to 31 December 2005 for 45 US states, representing over $95 \%$ of older people with dual eligibility living in the community in the USA. The study cohort was limited to patients with new antipsychotic treatment episodes, to avoid underascertainment of events that occurred soon after initiation of antipsychotic therapy. ${ }^{26,27}$ The study was approved by the Rutgers University institutional review board. New treatment episodes were defined by a claim for an antipsychotic drug immediately preceded by at least 180 days of eligibility without a claim for any antipsychotic medication or long-term nursing facility stay. At least one pharmacy claim and one Medicare service claim were required during the 180 days preceding the index date to ensure that patients were in contact with the healthcare system. All baseline variables were constructed from claims during the 180-day pre-index period. Patients were excluded if they had a claim for two or more different antipsychotic drugs at the index date, if the index claim for antipsychotic medication included no information regarding days of supply, or if the prescription was for an antipsychotic/ antidepressant combination product.

\section{Antipsychotic medications and follow-up}

Antipsychotic medications included risperidone, olanzapine, quetiapine, haloperidol, aripiprazole and ziprasidone. Other antipsychotics were used too infrequently to permit meaningful analysis. We constructed a calendar of antipsychotic exposure noting start of exposure, drug discontinuation and antipsychotic switch or augmentation. Antipsychotic discontinuation was defined as the last day of continuous supply, allowing for a gap of 7 days to account for late refills. Antipsychotic switch or augmentation was defined as a fill for an antipsychotic other than the index agent. Doses were converted into chlorpromazine equivalents (CPZeq) to assess dose effects across individual antipsychotics (see online Table DS1). ${ }^{28}$ Using this conversion approach, a dose of $2 \mathrm{mg}$ risperidone is approximately equivalent to $5 \mathrm{mg}$ olanzapine, $75 \mathrm{mg}$ quetiapine, $2 \mathrm{mg}$ haloperidol, $7.5 \mathrm{mg}$ aripiprazole or $60 \mathrm{mg}$ ziprasidone. An alternative conversion was used in sensitivity analyses. ${ }^{29}$

\section{Outcomes}

The primary outcome - non-cancer mortality within 180 days after the index date - was based on death date and cause of death information from the National Death Index. ${ }^{30,31}$ Cause-specific mortality was examined as a secondary outcome using broad diagnostic categories because misclassification of causes of death in older populations is likely: death from diseases of the circulatory system (ICD-10 codes I00-I99), ${ }^{32}$ death from cerebrovascular disease (ICD-10 I60-I69), death from diseases of the respiratory system (ICD-10 J00-J99) and death from other causes. $^{33}$

\section{Statistical analysis}

We first calculated sociodemographic, clinical and healthcare use characteristics at baseline for each of the prescribed antipsychotic subgroups, and estimated event rates for primary and secondary mortality outcomes with risperidone as the referent agent. To compare antipsychotics with respect to risk of non-cancer and cause-specific mortality, we performed crude and adjusted Cox proportional hazards regressions. Models were fitted at several levels of adjustment:

(a) unadjusted;

(b) adjusted for gender, ethnicity, age and calendar year; (c) adjusted for propensity score;

(d) adjusted for high-dimensional propensity score. ${ }^{34}$

Follow-up began from the day after the index antipsychotic claim and was censored at the day of discontinuation, antipsychotic switch or augmentation, 10 or more days in hospital, death, 180 days after index prescription claim, or end of study, whichever came first. Thirty days were added to the last day of follow-up for patients who discontinued antipsychotic therapy, to reduce potential bias from informative censoring if patients discontinued the drug because of adverse effects experienced shortly before death. Sensitivity analyses were performed without the 30-day addition. Propensity scores were calculated using a non-parsimonious logistic regression model including all variables presented in Tables 1 and 2. High-dimensional propensity scores were calculated using an automated algorithm that facilitated inclusion of many additional potential confounding variables. ${ }^{34}$ For all comparisons using traditional or high-dimensional propensity scores, propensity score distributions were plotted, evaluated for overlap and truncated at the margins of the propensity score distribution by $2.5 \%$ to ensure exclusion of patients who (based on their characteristics) would be expected to always receive one treatment over another. Propensity scores were included as deciles into the regression models. Analyses were stratified by dementia diagnosis and age group $(65-80$ years, $\geqslant 81$ years) to examine potential heterogeneity in mortality risk. In addition, we performed a dose-response analysis for all antipsychotics combined and for individual agents with sufficient sample sizes. For these analyses we constructed three dose strata based on the dose distributions observed for each individual antipsychotic and for all antipsychotics combined (online Table DS2). Lastly, we fitted dose-adjusted models that included antipsychotic dose ( $<50 \mathrm{mg}$ CPZeq, 50-99 mg CPZeq, 100-149 mg $\mathrm{CPZeq}, \geqslant 150 \mathrm{mg}$ CPZeq) and high-dimensional propensity score deciles. Dose-response and dose-adjusted analyses were based solely on antipsychotic index dose and did not consider dose changes over follow-up, as the majority of patients did not change dose between their first two antipsychotic fills $(88.2 \%)$ or between their first and last observed antipsychotic fill $(83.0 \%)$, and there was no meaningful difference between individual agents (online Table DS3).

\section{Results}

The study cohort comprised 136393 patients with new antipsychotic treatment episodes (Fig. 1). Risperidone was the most commonly used agent $(36.2 \%)$, followed by olanzapine $(32.5 \%)$, quetiapine (19.2\%), haloperidol (9.6\%), aripiprazole (1.4\%) and ziprasidone (1.1\%). Median starting doses were $0.5 \mathrm{mg}$ (risperidone), $5 \mathrm{mg}$ (olanzapine), $50 \mathrm{mg}$ (quetiapine), $1 \mathrm{mg}$ (haloperidol), $10 \mathrm{mg}$ (aripiprazole) and $40 \mathrm{mg}$ (ziprasidone). Mean time to censoring was 83 days. Censoring times were similar for the second-generation agents (Table 1), ranging from 77 days (ziprasidone) to 88 days (olanzapine) but were shorter for haloperidol (63 days). Censoring most commonly occurred owing to antipsychotic discontinuation $(83.8 \%)$ followed by day $180(13.2 \%)$ and death $(3.0 \%)$. The cohort was predominantly female, White and had a mean age of 79.1 years. Nearly a third of patients had a diagnosis of dementia. Although most baseline characteristics were broadly similar between individual agents, marked differences were evident in several characteristics. Most notably, patients beginning therapy with risperidone, haloperidol, quetiapine or ziprasidone had higher rates of diagnosed dementia than those starting olanzapine or aripiprazole. Diagnosed depression was highest among patients beginning therapy with aripiprazole, ziprasidone or quetiapine 
Patients $\geqslant 65$ years old who were eligible for both Medicare and Medicaid and began a new antipsychotic

treatment episode in the community

between 1 July 2001

and 31 December 2005 $n=575320$

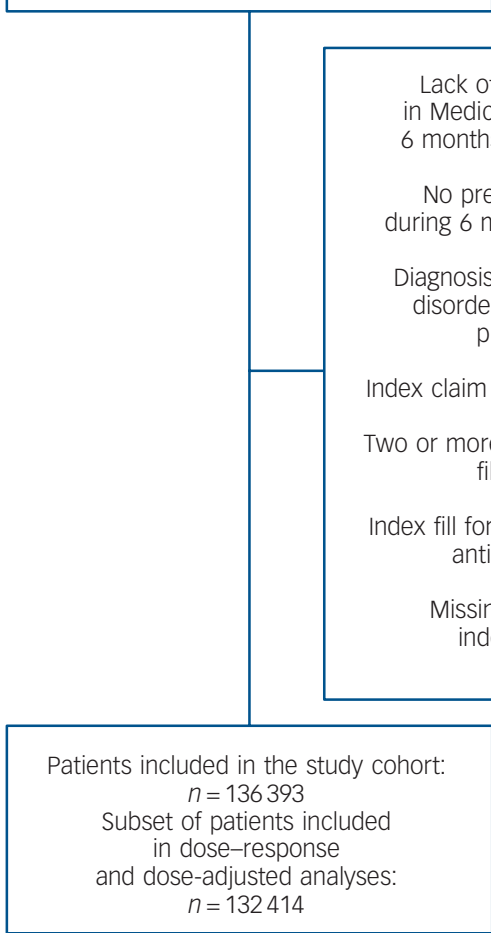

Fig. 1 Assembly of the study cohort.

and was relatively rare among patients starting haloperidol. Differences were also apparent in use of other psychotropic medications during the baseline period, with patients taking aripiprazole having the highest rate of use of other psychotropic medications and those taking haloperidol the lowest (Table 2).

\section{Mortality risk at 180 days}

Across all agents we observed 4216 non-cancer deaths in 31090 person-years of follow-up, for an overall non-cancer mortality rate of 13.6 per 100 person-years. An additional 180 cancer-related deaths were censored. Unadjusted event rates ranged from 31.4 (95\% CI 29.1-33.7) per 100 person-years for haloperidol to 5.8 (95\% CI 3.5-8.1) per 100 person-years for aripiprazole (online Table DS4). Hazard ratios (HRs) calculated at different levels of adjustment are presented in Table 3. In unadjusted comparisons with risperidone, haloperidol had a nearly doubled mortality risk, whereas aripiprazole, quetiapine, ziprasidone and olanzapine showed risk reductions ranging from 55\% (aripiprazole) to 22\% (olanzapine). Estimates for all comparisons moved monotonically towards the null with increasing levels of adjustment (Table 3). In analyses adjusted for high-dimensional propensity score, haloperidol demonstrated a $45 \%$ increase in risk and only quetiapine exhibited a statistically significant reduction in risk below risperidone (Fig. 2). The excess risk for haloperidol was greatest early after treatment initiation and attenuated during follow-up (online Table DS5). No significant difference from the overall results was observed for any of the specific causes of mortality (Table 3). Neither age- nor dementia-stratified models showed marked differences in the pattern of mortality risk between strata (online Table DS6).

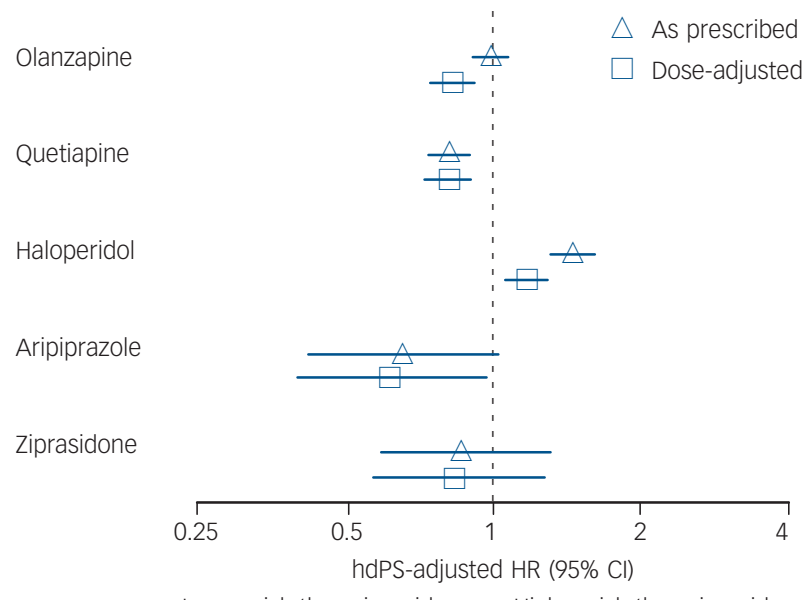

Fig. 2 Mortality hazard ratios for frequently used antipsychotic medications compared with risperidone as prescribed and adjusted for dose.

Risperidone is the referent in all comparisons; hdPS, high-dimensional propensity score; HR, hazard ratio. Dose adjustment was performed with the following chlorpromazine equivalent dose categories: $<50 \mathrm{mg}$ 50-99 mg 100-149 mg $\geqslant 150 \mathrm{mg}$.

\section{Dose-response and dose-adjusted analyses}

For all study antipsychotics combined, a dose-response relationship was apparent with hazard ratios of 1.36 (95\% CI 1.24-1.49) for high $v$. low dose and 1.19 (95\% CI 1.10-1.27) for medium $v$. low dose (Fig. 3). Among the four most widely used agents, all but quetiapine showed significant dose response. Haloperidol demonstrated the strongest dose-response effect. Aripiprazole and ziprasidone were not used with sufficient frequency to permit meaningful evaluation of dose-response risk of mortality.

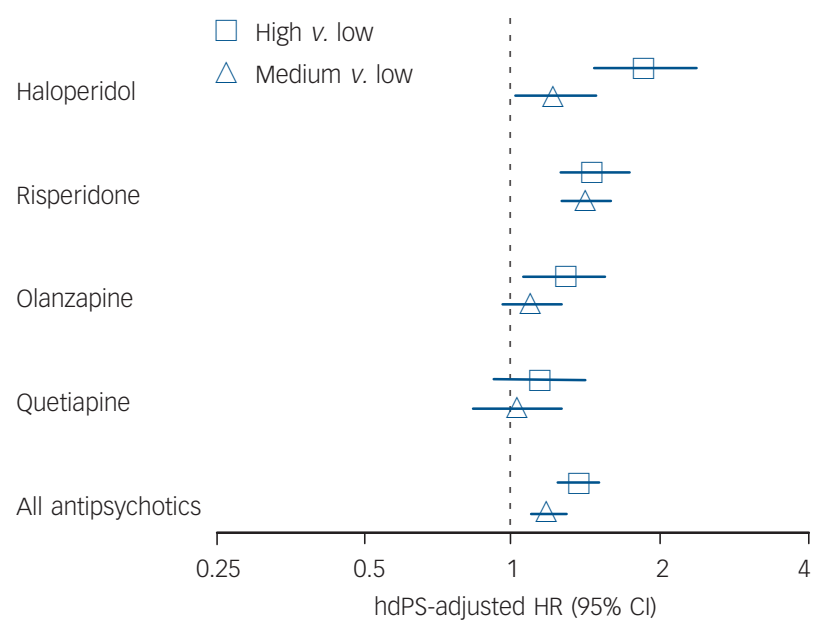

Lower risk than low dose Higher risk than low dose

Fig. 3 Dose-response analysis for antipsychotic medications and non-cancer mortality.

Analyses were restricted to patients taking solid oral dosage forms of the antipsychotic drug $(n=132414)$. The low-dose group of each agent served as the reference group for each comparison. Owing to insufficient event numbers in individual dose strata, no result is presented for aripiprazole or ziprasidone. The following dose ranges were used: haloperidol low $\leqslant 1 \mathrm{mg}$, medium $>1-4 \mathrm{mg}$, high $>4 \mathrm{mg}$; risperidone low $\leqslant 0.5 \mathrm{mg}$, medium $>0.5-1 \mathrm{mg}$, high $>1 \mathrm{mg}$; olanzapine low $\leqslant 2.5 \mathrm{mg}$, medium $>2.5-5 \mathrm{mg}$, high $>5 \mathrm{mg}$; quetiapine low $\leqslant 25 \mathrm{mg}$, medium $>25-50 \mathrm{mg}$, high > $>50 \mathrm{mg}$; all antipsychotics (in chlorpromazine equivalents) low $<50$ mg CPZeq, medium 50-75 mg CPZeq, high > 75 mg CPZeq. hdPS, high-dimensional propensity score; HR, hazard ratio. 


\begin{tabular}{|c|c|c|c|c|c|c|c|}
\hline & Full cohort & Risperidone & Olanzapine & Quetiapine & Haloperidol & Aripiprazole & Ziprasidone \\
\hline Patients, $n$ (\%) & $136393(100.0)$ & $49333(36.2)$ & $44296(32.5)$ & $26130(19.2)$ & $13159(9.6)$ & $1908(1.4)$ & 1567 (1.1) \\
\hline \multicolumn{8}{|l|}{ Dose (CPZeq), $n$ (\%) ${ }^{a}$} \\
\hline$<50 \mathrm{mg}$ & $43821(32.1)$ & $27639(56.0)$ & 577 (1.3) & 12519 (47.9) & $2582(19.6)$ & $116(6.1)$ & $388(24.8)$ \\
\hline 50-99 mg & $49470(36.3)$ & $14391(29.2)$ & $20669(46.7)$ & $8523(32.6)$ & $4564(34.7)$ & $696(36.5)$ & $627(40.0)$ \\
\hline $100-149 \mathrm{mg}$ & 24185 (17.7) & $3907(7.9)$ & $14272(32.2)$ & $2797(10.7)$ & $2269(17.2)$ & $602(31.6)$ & $338(21.6)$ \\
\hline$\geqslant 150 \mathrm{mg}$ & $14912(10.9)$ & $1793(3.6)$ & $7710(17.4)$ & $1904(7.3)$ & $2863(21.8)$ & $471(24.7)$ & $171(10.9)$ \\
\hline Time to censoring, days: mean (s.d.) & $83.3(51.7)$ & $83.4(52.2)$ & $88.0(51.2)$ & $85.8(53.9)$ & $62.8(40.7)$ & $82.1(59.8)$ & $76.5(48.8)$ \\
\hline \multicolumn{8}{|l|}{ Year of index date, $n$ (\%) } \\
\hline 2001 & $15513(11.4)$ & $5973(12.1)$ & $5688(12.8)$ & $1445(5.5)$ & $2327(17.7)$ & $N A^{b}$ & $80(5.1)$ \\
\hline 2002 & $31963(23.4)$ & $11882(24.1)$ & $13313(30.1)$ & $3306(12.7)$ & $3264(24.8)$ & $N A^{b}$ & $189(12.1)$ \\
\hline 2003 & $32520(23.8)$ & $11227(22.8)$ & $12534(28.3)$ & $5301(20.3)$ & $2715(20.6)$ & $383(20.1)$ & $360(23.0)$ \\
\hline 2004 & $30583(22.4)$ & $11381(23.1)$ & $7978(18.0)$ & $7615(29.1)$ & $2441(18.6)$ & $696(36.5)$ & $472(30.1)$ \\
\hline 2005 & $25814(18.9)$ & $8870(18.0)$ & $4783(10.8)$ & $8463(32.4)$ & $2412(18.3)$ & $820(43.0)$ & $466(29.7)$ \\
\hline \multicolumn{8}{|l|}{ Demographic characteristics } \\
\hline Male, $n(\%)$ & $32521(23.8)$ & $11114(22.5)$ & $10643(24.0)$ & $6622(25.3)$ & $3291(25.0)$ & $473(24.8)$ & $378(24.1)$ \\
\hline Age, years: mean (s.d.) & $79.1(8.3)$ & $80.0(8.3)$ & $78.3(8.2)$ & $77.8(8.1)$ & $81.8(8.3)$ & $75.9(7.6)$ & $76.7(7.9)$ \\
\hline \multicolumn{8}{|l|}{ Age group, $n(\%)$} \\
\hline $65-70$ & $25486(18.7)$ & 7753 (15.7) & $9293(21.0)$ & $5970(22.9)$ & $1446(11.0)$ & $580(30.4)$ & $444(28.3)$ \\
\hline $71-75$ & 24209 (17.8) & 7948 (16.1) & $8614(19.5)$ & $5152(19.7)$ & $1788(13.6)$ & 407 (21.3) & 300 (19.1) \\
\hline $76-80$ & $27376(20.1)$ & $9864(20.0)$ & 8973 (20.3) & $5251(20.1)$ & $2551(19.4)$ & 397 (20.8) & $340(21.7)$ \\
\hline $81-85$ & $25917(19.0)$ & $10028(20.3)$ & 7933 (17.9) & $4701(18.0)$ & $2735(20.8)$ & $274(14.4)$ & $246(15.7)$ \\
\hline $86-90$ & $19664(14.4)$ & 7993 (16.2) & 5817 (13.1) & $3073(11.8)$ & $2462(18.7)$ & $168(8.8)$ & $151(9.6)$ \\
\hline $91-95$ & $10747(7.9)$ & $4429(9.0)$ & $2973(6.7)$ & $1577(6.0)$ & 1630 (12.4) & $72(3.8)$ & $66(4.2)$ \\
\hline $96-100$ & $2994(2.2)$ & $1318(2.7)$ & $693(1.6)$ & $406(1.6)$ & $547(4.2)$ & $N A^{b}$ & $20(1.3)$ \\
\hline \multicolumn{8}{|l|}{ Ethnicity, $n$ (\%) } \\
\hline White & $73730(54.1)$ & $26393(53.5)$ & 24419 (55.1) & $14734(56.4)$ & $6346(48.2)$ & 1009 (52.9) & 829 (52.9) \\
\hline African American & $22620(16.6)$ & 9405 (19.1) & 5899 (13.3) & 3609 (13.8) & $3126(23.8)$ & $271(14.2)$ & $310(19.8)$ \\
\hline Hispanic & $12811(9.4)$ & $4336(8.8)$ & $3883(8.8)$ & $2981(11.4)$ & $1168(8.9)$ & $276(14.5)$ & $167(10.7)$ \\
\hline Other/unknown & $27232(20.0)$ & 9199 (18.7) & 10095 (22.8) & $4806(18.4)$ & 2519 (19.1) & $352(18.5)$ & $261(16.7)$ \\
\hline
\end{tabular}

After conversion to chlorpromazine equivalents, marked differences in dose distributions were observed among agents (Tables 1 and DS2). Most notably, 56\% and 48\% of risperidone and quetiapine use respectively were at doses below $50 \mathrm{mg}$ CPZeq, with significantly lower proportions for olanzapine (1\%), aripiprazole (6\%) and haloperidol (20\%). Conversely, use at doses of $100 \mathrm{mg}$ CPZeq or above was significantly more common for aripiprazole (56\%), olanzapine (50\%) and haloperidol (39\%) compared with risperidone $(11.5 \%)$ and quetiapine (18\%). At the low end of the spectrum these differences are largely attributable to differences in the lowest available tablet strength, e.g. $0.25 \mathrm{mg}$ ( $12.5 \mathrm{mg}$ CPZeq) for risperidone and $2.5 \mathrm{mg}(50 \mathrm{mg}$ CPZeq) for olanzapine. In analyses controlled for CPZeq dose categories and high-dimensional propensity scores, haloperidol was associated with an $18 \%$ (95\% CI 6-33) increase in risk, whereas aripiprazole ( $\mathrm{HR}=0.62,95 \% \mathrm{CI} 0.40-0.97)$, quetiapine $(\mathrm{HR}=0.80,95 \% \mathrm{CI} 0.72-0.89)$ and olanzapine $(\mathrm{HR}=0.82,95 \%$ CI $0.74-0.90)$ had appreciably decreased non-cancer mortality risks (Fig. 2). Point estimates remained essentially unchanged following restriction to patients initially taking doses commonly used for both comparators (data not shown). Dose-response and dose-adjusted findings using an alternative CPZeq conversion algorithm were consistent with results shown above (data not shown). ${ }^{29}$

\section{Discussion}

In a large, community-based cohort of people over 65 years old, significant differences were evident in mortality risk during the first 6 months of treatment with commonly prescribed antipsychotics. As prescribed and as compared with risperidone, mortality risk was substantially higher for haloperidol and lower for quetiapine. No marked difference in mortality risk was observed between risperidone and olanzapine. Confidence intervals for aripiprazole and ziprasidone were wide owing to infrequent use of these drugs during the study period, warranting further study of their comparative mortality risks in older adults. Dose-response in mortality risk was observed for haloperidol, risperidone and olanzapine, but not for quetiapine. Adjusting for dose in the analysis markedly reduced the magnitude of excess mortality risk observed for haloperidol and lowered the risk associated with olanzapine. Stratified analyses provided no evidence of treatment effect heterogeneity by age group or presence of dementia.

In several respects our results confirm those from previous studies that directly compared individual antipsychotic agents. ${ }^{23,24}$ Hazard ratio point estimates for comparisons with risperidone across the three studies ranged from 1.45 to 1.81 for haloperidol, from 0.99 to 1.06 for olanzapine and from 0.74 to 0.83 for quetiapine. Indirect contrasts based on comparisons of individual antipsychotics with non-use showed similar findings. ${ }^{25}$ Aripiprazole and ziprasidone were only examined in one previous study, ${ }^{23}$ where no differences in mortality risk in relation to risperidone were observed. It may be noted that the higher risk observed for haloperidol in this study $(\mathrm{HR}=1.81)$ compared with our community study $(\mathrm{HR}=1.45)$ might be the result of higher haloperidol doses prescribed in nursing homes. Whereas $21.7 \%$ of initial haloperidol prescriptions in the nursing home were over $4 \mathrm{mg}$, only $13.9 \%$ were over $4 \mathrm{mg}$ in the community-based cohort. ${ }^{23}$ An increased mortality risk for haloperidol compared with risperidone is also broadly consistent with reports of greater mortality risk associated with first- $v$. second-generation 


\begin{tabular}{|c|c|c|c|c|c|c|c|}
\hline & $\begin{array}{l}\text { Full cohort } \\
n=136393\end{array}$ & $\begin{array}{c}\text { Risperidone } \\
n=49333\end{array}$ & $\begin{array}{l}\text { Olanzapine } \\
n=44296\end{array}$ & $\begin{array}{l}\text { Quetiapine } \\
n=26130\end{array}$ & $\begin{array}{l}\text { Haloperidol } \\
n=13159\end{array}$ & $\begin{array}{l}\text { Aripiprazole } \\
n=1908\end{array}$ & $\begin{array}{l}\text { Ziprasidone } \\
n=1567\end{array}$ \\
\hline \multicolumn{8}{|l|}{ Psychiatric morbidity, n (\%) } \\
\hline Dementia & $43183(31.7)$ & $17543(35.6)$ & $11400(25.7)$ & $8518(32.6)$ & 4676 (35.5) & $538(28.2)$ & $508(32.4)$ \\
\hline Depression & $26523(19.5)$ & $8939(18.1)$ & $8637(19.5)$ & $6671(25.5)$ & $1118(8.5)$ & $706(37.0)$ & $452(28.8)$ \\
\hline Anxiety & $4049(3.0)$ & $1322(2.7)$ & $1341(3.0)$ & $960(3.7)$ & $286(2.2)$ & $75(3.9)$ & $65(4.2)$ \\
\hline Delirium & $6949(5.1)$ & $2612(5.3)$ & $1734(3.9)$ & $1661(6.4)$ & $713(5.4)$ & $116(6.1)$ & $113(7.2)$ \\
\hline Non-schizophrenia psychosis ${ }^{a}$ & $11866(8.7)$ & $4859(9.9)$ & $3126(7.1)$ & $2345(9.0)$ & $1039(7.9)$ & $274(14.4)$ & $223(14.2)$ \\
\hline \multicolumn{8}{|l|}{ Cardiovascular morbidity, $n$ (\%) } \\
\hline Myocardial infarction & $6467(4.7)$ & $2518(5.1)$ & $1698(3.8)$ & $1281(4.9)$ & $822(6.3)$ & $68(3.6)$ & $80(5.1)$ \\
\hline Arrhythmias & $10897(8.0)$ & $3901(7.9)$ & $3481(7.9)$ & $2015(7.7)$ & $1254(9.5)$ & $128(6.7)$ & $118(7.5)$ \\
\hline Ischaemic heart disease & $9073(6.7)$ & $3166(6.4)$ & $3057(6.9)$ & $1780(6.8)$ & $884(6.7)$ & $95(5.0)$ & $91(5.8)$ \\
\hline Hypertension & $84747(62.1)$ & 30615 (62.1) & $27285(61.6)$ & $16590(63.5)$ & $8011(60.9)$ & $1233(64.6)$ & $1013(64.7)$ \\
\hline Congestive heart failure & $26208(19.2)$ & $9863(20.0)$ & 7766 (17.5) & 4745 (18.2) & $3245(24.7)$ & $315(16.5)$ & $274(17.5)$ \\
\hline Cerebrovascular disease, $n(\%)$ & $23703(17.4)$ & $8912(18.1)$ & $6842(15.5)$ & $4621(17.7)$ & $2769(21.0)$ & $288(15.1)$ & $271(17.3)$ \\
\hline \multicolumn{8}{|l|}{ Other comorbidities, $n$ (\%) } \\
\hline Diabetes & $40043(29.4)$ & $14844(30.1)$ & $12169(27.5)$ & $7874(30.1)$ & $4034(30.7)$ & $634(33.2)$ & $488(31.1)$ \\
\hline Parkinson's disease & $5201(3.8)$ & $1312(2.7)$ & $1234(2.8)$ & $2186(8.4)$ & $354(2.7)$ & $56(2.9)$ & $59(3.8)$ \\
\hline \multicolumn{8}{|l|}{$\begin{array}{l}\text { General indicators of comorbidity } \\
\text { Charlson comorbidity index }\end{array}$} \\
\hline $0-1$ & $54409(39.9)$ & $19141(38.8)$ & $18551(41.9)$ & $10494(40.2)$ & 4764 (36.2) & $786(41.2)$ & $673(43.0)$ \\
\hline $2-5$ & 71807 (52.7) & $26441(53.6)$ & 22831 (51.5) & 13653 (52.3) & $7134(54.2)$ & $972(50.9)$ & 776 (49.5) \\
\hline$>5$ & $10177(7.5)$ & $3751(7.6)$ & $2914(6.6)$ & $1983(7.6)$ & $1261(9.6)$ & $150(7.9)$ & $118(7.5)$ \\
\hline $\begin{array}{l}\text { Number of different prescription } \\
\text { drugs received, mean (s.d.) }\end{array}$ & $12.4(6.7)$ & 119 (6 5) & $127(67)$ & $132(70)$ & $114(64)$ & $135(69)$ & $127(66)$ \\
\hline Number of out-patient visits, & 12.70 .17 & ) & $12.1(0.1)$ & $(10.2(1.0)$ & $11.4(0.4)$ & (10.0 & (1).1 \\
\hline mean (s.d.) & $2.2(3.8)$ & $2.2(3.6)$ & $2.2(3.8)$ & $2.5(4.1)$ & $2.2(3.5)$ & $2.2(3.8)$ & $2.2(3.4)$ \\
\hline $\begin{array}{l}\text { Time in hospital, days: } \\
\text { mean (s.d.) }\end{array}$ & $5.3(12.4)$ & $5.6(12.8)$ & $4.6(11.4)$ & $5.7(13.2)$ & $5.9(12.4)$ & $5.5(12.0)$ & $7.2(14.5)$ \\
\hline \multicolumn{8}{|l|}{$\begin{array}{l}\text { History of psychotropic } \\
\text { prescriptions, } n(\%)\end{array}$} \\
\hline Antidepressants & $67928(49.8)$ & 23369 (47.4) & $22833(51.6)$ & $15051(57.6)$ & 4526 (34.4) & $1241(65.0)$ & $908(58.0)$ \\
\hline Hypnotic agents & $58386(42.8)$ & $19443(39.4)$ & $19694(44.5)$ & $12604(48.2)$ & $4933(37.5)$ & $963(50.5)$ & $749(47.8)$ \\
\hline Other psychoactive agents ${ }^{c}$ & $10036(7.4)$ & $3354(6.8)$ & $3412(7.7)$ & $2187(8.4)$ & $740(5.6)$ & $202(10.6)$ & $141(9.0)$ \\
\hline Dementia medication & $33720(24.7)$ & $13384(27.1)$ & $9231(20.8)$ & $7338(28.1)$ & $2835(21.5)$ & $503(26.4)$ & $429(27.4)$ \\
\hline
\end{tabular}

antipsychotics. ${ }^{2,9-12}$ Taken together, these findings suggest that at the doses used in clinical practice differences in mortality risks between individual agents are largely consistent across diverse populations of older adults.

Consistent with the nursing-home study, higher as compared with lower dosing tends to carry greater mortality risk for older people living in the community. ${ }^{23}$ Higher risk of mortality is associated with higher doses of risperidone, olanzapine and haloperidol, but not quetiapine. Potential pharmacological explanations for the absence of a dose-response relationship for quetiapine, which was observed in both studies, remain incompletely understood. ${ }^{35,36}$ Because dose-response analyses in both studies were empirically constructed based on each agent's observed dose distribution, a simple explanation for the lack of dose response for quetiapine is that there is less variation in clinical quetiapine dosing than for most other antipsychotics (Table DS2). As greater clinical experience accumulates with aripiprazole and ziprasidone in older patients, it will be important to assess the dose-related mortality risks of these medications.

Adjustment for dose in the analysis markedly altered the magnitude - and for some medications the direction - of the individual agent mortality effect estimates. Assuming the validity of the dose equivalencies used in our study, this suggests that mortality differences between antipsychotics are partially a function of dosing differences in clinical practice rather than the pharmacodynamic properties of individual agents. Particularly, the excess mortality risk of haloperidol compared with risperidone may be at least partially due to more aggressive dosing. Previous studies did not report significant changes in their findings after dose adjustment. ${ }^{23,24}$ The differences among studies in individual agent dose-adjusted mortality effect estimates could be due to differences in dose-adjustment methods or patient populations. ${ }^{23,24}$

No substantial comparative risk difference between patient groups with and without clinical dementia diagnoses was evident in this or in our companion nursing-home study. ${ }^{23}$ The current regulatory advisories specifically relate to the treatment of elderly patients with dementia. ${ }^{14,15,19}$ In light of the widespread use of antipsychotics in the out-patient treatment of older people without a dementia diagnosis, safety considerations specific to this population are important. ${ }^{37}$ Because the relative risks of antipsychotic-related mortality in older adults appear to be similar in out-patient groups with and without a dementia diagnosis, concerns raised by the regulatory advisories may apply equally to older patients without dementia. Although our study did not include an untreated comparison group and therefore cannot directly address the question whether antipsychotics increase mortality risk in patients without dementia compared with no treatment, the dose-response findings in mortality risk are consistent with an increased absolute mortality risk associated with antipsychotic medications. 


\begin{tabular}{|c|c|c|c|c|}
\hline & \multicolumn{4}{|c|}{ Hazard ratio $(95 \% \mathrm{Cl})$} \\
\hline & \multirow[t]{2}{*}{ Unadjusted } & \multicolumn{3}{|c|}{ Adjusted } \\
\hline & & Age, gender, ethnicity and year & Propensity score & High-dimensional propensity score \\
\hline \multicolumn{5}{|c|}{ Non-cancer mortality } \\
\hline Risperidone & 1.0 & 1.0 & 1.0 & 1.0 \\
\hline Olanzapine & $0.78(0.72-0.84)$ & $0.87(0.80-0.94)$ & $0.91(0.84-0.98)$ & $0.99(0.91-1.07)$ \\
\hline Quetiapine & $0.66(0.60-0.73)$ & $0.71(0.64-0.78)$ & $0.75(0.68-0.83)$ & $0.81(0.73-0.89)$ \\
\hline Haloperidol & $1.97(1.79-2.16)$ & $1.88(1.71-2.06)$ & $1.63(1.48-1.79)$ & $1.45(1.31-1.60)$ \\
\hline Aripiprazole & $0.45(0.30-0.67)$ & $0.54(0.36-0.81)$ & $0.60(0.40-0.89)$ & $0.66(0.42-1.02)$ \\
\hline Ziprasidone & $0.64(0.44-0.93)$ & $0.74(0.51-1.09)$ & $0.75(0.51-1.11)$ & $0.87(0.59-1.30)$ \\
\hline \multicolumn{5}{|c|}{ Cause-specific mortality } \\
\hline \multicolumn{5}{|c|}{ Circulatory system } \\
\hline Risperidone & 1.0 & 1.0 & 1.0 & 1.0 \\
\hline Olanzapine & $0.83(0.75-0.92)$ & $0.93(0.84-1.04)$ & $0.97(0.88-1.08)$ & $1.06(0.95-1.18)$ \\
\hline Quetiapine & $0.68(0.60-0.78)$ & $0.74(0.64-0.84)$ & $0.81(0.70-0.92)$ & $0.83(0.72-0.95)$ \\
\hline Haloperidol & $2.02(1.79-2.29)$ & $1.89(1.67-2.15)$ & $1.63(1.43-1.85)$ & $1.45(1.26-1.65)$ \\
\hline Aripiprazole & $0.45(0.26-0.78)$ & $0.56(0.32-0.98)$ & $0.63(0.37-1.10)$ & $0.70(0.38-1.28)$ \\
\hline Ziprasidone & $0.70(0.43-1.15)$ & $0.84(0.51-1.37)$ & $0.89(0.53-1.45)$ & $0.92(0.53-1.59)$ \\
\hline \multicolumn{5}{|c|}{ Cerebrovascular disease } \\
\hline Risperidone & 1.0 & 1.0 & 1.0 & 1.0 \\
\hline Olanzapine & $0.73(0.56-0.94)$ & $0.82(0.63-1.07)$ & $0.86(0.66-1.12)$ & $0.97(0.74-1.27)$ \\
\hline Quetiapine & $0.64(0.46-0.88)$ & $0.72(0.51-1.00)$ & $0.80(0.57-1.12)$ & $0.87(0.62-1.23)$ \\
\hline Haloperidol & $2.23(1.66-3.00)$ & $2.07(1.53-2.78)$ & $1.76(1.30-2.38)$ & $1.61(1.17-2.20)$ \\
\hline Aripiprazole & $0.20(0.03-1.45)$ & $0.27(0.04-1.94)$ & $0.33(0.05-2.35)$ & $0.36(0.05-2.59)$ \\
\hline Ziprasidone & $0.50(0.12-2.01)$ & $0.62(0.15-2.51)$ & $0.62(0.15-2.50)$ & $0.37(0.05-2.66)$ \\
\hline \multicolumn{5}{|c|}{ Respiratory system } \\
\hline Risperidone & 1.0 & 1.0 & 1.0 & 1.0 \\
\hline Olanzapine & $0.81(0.66-0.99)$ & $0.85(0.69-1.04)$ & $0.86(0.70-1.05)$ & $0.90(0.73-1.11)$ \\
\hline Quetiapine & $0.67(0.52-0.87)$ & $0.71(0.54-0.92)$ & $0.71(0.55-0.93)$ & $0.85(0.65-1.11)$ \\
\hline Haloperidol & $1.88(1.46-2.40)$ & $1.87(1.45-2.40)$ & $1.63(1.26-2.10)$ & $1.38(1.06-1.79)$ \\
\hline Aripiprazole & $0.80(0.35-1.79)$ & $0.97(0.43-2.20)$ & $0.94(0.42-2.14)$ & $1.51(0.65-3.52)$ \\
\hline Ziprasidone & $0.34(0.08-1.35)$ & $0.38(0.09-1.54)$ & $0.36(0.09-1.47)$ & $0.47(0.12-1.91)$ \\
\hline \multicolumn{5}{|l|}{ Other } \\
\hline Risperidone & 1.0 & 1.0 & 1.0 & 1.0 \\
\hline Olanzapine & $0.69(0.60-0.80)$ & $0.77(0.67-0.89)$ & $0.81(0.70-0.93)$ & $0.87(0.75-1.01)$ \\
\hline Quetiapine & $0.62(0.52-0.74)$ & $0.66(0.55-0.79)$ & $0.69(0.57-0.82)$ & $0.71(0.59-0.86)$ \\
\hline Haloperidol & $1.91(1.61-2.26)$ & $1.85(1.56-2.19)$ & $1.63(1.37-1.93)$ & $1.46(1.22-1.74)$ \\
\hline Aripiprazole & $0.28(0.12-0.68)$ & $0.33(0.13-0.79)$ & $0.37(0.15-0.90)$ & $0.45(0.18-1.09)$ \\
\hline Ziprasidone & $0.66(0.34-1.28)$ & $0.75(0.39-1.46)$ & $0.74(0.38-1.44)$ & $0.89(0.46-1.73)$ \\
\hline
\end{tabular}

\section{Study limitations}

Our study is subject to several limitations. First, residual confounding is a potential source of bias if unobserved variables affect antipsychotic choice, dose and risk of mortality. Severity of dementia and dementia-related behavioural symptoms, which are not directly observable in claims data, may have confounded the observed associations. However, our companion nursinghome study, which had access to clinical measures of dementia severity and behavioural symptoms from nursing staff ratings, did not observe changes in mortality estimates when these ratings were added to the propensity score models. ${ }^{38}$ Second, we aimed to estimate comparative mortality risks for antipsychotics at clinically equivalent doses by adjusting for chlorpromazineequivalent dose categories. However, the chlorpromazine dose equivalency schedule, which was developed for schizophrenia treatment, has uncertain applicability to the lower antipsychotic doses used in older patients without psychosis. The dose-adjusted findings in our study thus have to be interpreted with appropriate caution. Nevertheless, our findings were robust using two different conversion algorithms. ${ }^{28,29}$ Third, dose analyses were based on the antipsychotic dose of the index prescription and might therefore have introduced dose misclassification if titration schedules varied across agents. Because dose changes during follow-up occurred in only a small minority of patients, the effects of such misclassification are likely to be limited. Fourth, considerable uncertainty exists about the intended clinical indications for antipsychotic treatment in our study population. Although patients with schizophrenia, bipolar disorder or cancer were excluded, only about a third of patients had a clinical diagnosis of dementia. Underascertainment of dementia in claims data is well recognised, ${ }^{39,40}$ resulting from both underdiagnosis of dementia in clinical practice (particularly in the early stage of the disease, where diagnostic sensitivity has been estimated at $9-41 \%),{ }^{41}$ and incomplete coding of diagnosed dementia. ${ }^{42}$ It is thus likely that considerably more than a third of the patients in our study had at least early-stage dementia. Fifth, our study was limited to comparisons between antipsychotic agents commonly used in the USA during the years 2001-2005 and therefore could not provide estimates of the comparative mortality risk associated with newer agents or agents not approved in the USA such as amisulpride. Finally, our results are limited to the first 6 months of treatment and therefore do not inform the long-term comparative safety of antipsychotic treatment, and no comparison is presented with patients not receiving antipsychotic medication.

\section{Implications}

Clinicians treating older people with dementia should balance evidence of mortality risks and other adverse effects with efficacy in managing disruptive and potentially dangerous behaviour. ${ }^{4,43-45}$ 
Generally, antipsychotics should be reserved for patients with psychotic and severe mood symptoms that cause significant distress and to situations with immediate risk of harm to the patient or others, and should be avoided as a treatment of cognitive deficits. Treatment with antipsychotics should target specific symptoms, be initiated at low dose and used for short periods, and should not be initiated until medications and alternative treatable medical conditions that might cause or exacerbate behavioural symptoms have been excluded. ${ }^{45,46}$ For many patients non-pharmacological options may offer a safer treatment alternative but the evidence base for these interventions remains underdeveloped and barriers exist to their widespread implementation. ${ }^{4-49}$ If antipsychotic therapy is initiated the choice of medication should be guided by patient preferences and predisposition regarding these agents' adverse effects profiles including their comparative mortality risks, ${ }^{45}$ as well as each agent's efficacy for the targeted symptom. For global behavioural symptoms in dementia, aripiprazole, olanzapine and risperidone show similar small but statistically significant benefits, with less robust evidence for the benefit of quetiapine. ${ }^{4}$

Current efforts in both the UK and the USA aim to reduce antipsychotic prescribing in older adults without severe mental illness. ${ }^{50,51}$ However, because antipsychotic use in this population is likely to remain significant, evidence regarding the comparative safety of these agents remains important. The comparative pharmacoepidemiological safety studies to date paint a largely consistent picture regarding the comparative mortality risks of the most widely used antipsychotic agents, particularly at doses commonly used in clinical practice. ${ }^{23,24}$ Because the newer agents aripiprazole and ziprasidone were less commonly used during the study periods, greater uncertainty remains about the comparative risks of these agents and particularly their dose-response profiles.

This study suggests that dose adjustment markedly affects the comparative mortality risks associated with individual agents. Comparative data on the effectiveness of individual agents across the dose spectrum is therefore needed to better inform benefitrisk assessment and agent selection. Even in the absence of such data, current guidelines supported by the dose-response findings of this and other observational studies suggest that if in specific cases where the potential benefit is likely to outweigh the adverse effects the decision to use antipsychotic medications is made, it is critical to select the smallest dose effective for the targeted behaviour through careful dose titration, especially for haloperidol. ${ }^{43,46,52}$

\footnotetext{
Tobias Gerhard, PhD, Institute for Health, Health Care Policy and Aging Research, Rutgers University, New Brunswick, New Jersey, and Department of Pharmacy Practice and Administration, Ernest Mario School of Pharmacy, Rutgers University, Piscataway, New Jersey; Krista Huybrechts, PhD, Division of Pharmacoepidemiology and Pharmacoeconomics, Department of Medicine, Brigham and Women's Hospital and Harvard Medical School, Boston, Massachusetts; Mark olfson, MD MPH, Department of Psychiatry, College of Physicians and Surgeons, Columbia University, and the New York State Psychiatric Institute, New York, New York; Sebastian Schneeweiss, MD, SCD, Division of Pharmacoepidemiology and Sebastian Schneeweiss, MD, SCD, Division of Pharmacoepidemiology and
Pharmacoeconomics, Department of Medicine, Brigham and Women's Hospital and Pharmacoeconomics, Department of Medicine, Brigham and Women's Hospital and
Harvard Medical School, Boston, Massachusetts; William V. Bobo, MD, Department of Psychiatry, Vanderbilt University School of Medicine, Nashville, Tennessee; P. Murali Doraiswamy, MD, Department of Psychiatry, Duke University Medical Center, Durham, North Carolina; D. P. Devanand, MD, Department of Psychiatry, College of Physicians and Surgeons, Columbia University, and the New York State Psychiatric Institute, New York, New York; Judith A. Lucas, EdD RN, Cecilia Huang PhD, Edmond S. Malka, PhD, MPH, Institute for Health, Health Care Policy and Aging Research, Rutgers University, New Brunswick, New Jersey; Raisa Levin, MS Division of Pharmacoepidemiology and Pharmacoeconomics, Department of Medicine, Brigham and Women's Hospital and Harvard Medical School, Boston, Massachusetts; Stephen Crystal, PhD, Institute for Health, Health Care Policy and
} Aging Research, Rutgers University, New Brunswick, New Jersey, USA

Correspondence: Professor Tobias Gerhard, Ernest Mario School of Pharmacy, Rutgers University, 112 Paterson Street, New Brunswick, NJ 08901, USA. Email: tgerhard@rci.rutgers.edu

First received 7 Jan 2013, final revision 29 Apr 2013, accepted 8 May 2013

\section{Funding}

This work was supported by Agency for Healthcare Research and Quality/Food and Drug Administration (AHRQ/FDA) award HS017918 and AHRQ award HS016097.

\section{References}

1 Schneider LS, Dagerman KS, Insel P. Risk of death with atypical antipsychotic drug treatment for dementia: meta-analysis of randomized placebocontrolled trials. JAMA 2005; 294: 1934-43.

2 Wang PS, Schneeweiss S, Avorn J, Fischer MA, Mogun H, Solomon DH, et al. Risk of death in elderly users of conventional vs. atypical antipsychotic medications. N Engl J Med 2005; 353: 2335-41.

3 Schneider LS, Dagerman K, Insel PS. Efficacy and adverse effects of atypical antipsychotics for dementia: meta-analysis of randomized, placebo-controlled trials. Am J Geriatr Psychiatry 2006; 14: 191-210.

4 Maher AR, Maglione M, Bagley S, Suttorp M, Hu JH, Ewing B, et al. Efficacy and comparative effectiveness of atypical antipsychotic medications for off-label uses in adults: a systematic review and meta-analysis. JAMA 2011; 306: 1359-69.

5 Ballard C, Creese B, Corbett A, Aarsland D. Atypical antipsychotics for the treatment of behavioral and psychological symptoms in dementia, with a particular focus on longer term outcomes and mortality. Expert Opin Drug Saf 2011; 10: 35-43.

6 Ballard C, Howard R. Neuroleptic drugs in dementia: benefits and harm. Nat Rev Neurosci 2006; 7: 492-500.

7 Crystal S, Olfson M, Huang C, Pincus H, Gerhard T. Broadened use of atypical antipsychotics: safety, effectiveness, and policy challenges. Health Aff (Millwood) 2009; 28: w770-81.

8 Shah SM, Carey IM, Harris T, Dewilde S, Cook DG. Antipsychotic prescribing to older people living in care homes and the community in England and Wales. Int J Geriatr Psychiatry 2011; 26: 423-34.

9 Gill SS, Bronskill SE, Normand SL, Anderson GM, Sykora K, Lam K, et al. Antipsychotic drug use and mortality in older adults with dementia. Ann Intern Med 2007; 146: 775-86.

10 Schneeweiss S, Setoguchi S, Brookhart A, Dormuth C, Wang PS. Risk of death associated with the use of conventional versus atypical antipsychotic drugs among elderly patients. CMAJ 2007; 176: 627-32.

11 Liperoti R, Onder G, Landi F, Lapane KL, Mor V, Bernabei R, et al. All-cause mortality associated with atypical and conventional antipsychotics among nursing home residents with dementia: a retrospective cohort study. J Clin Psychiatry 2009; 70: 1340-7.

12 Rochon PA, Normand SL, Gomes T, Gill SS, Anderson GM, Melo M, et al. Antipsychotic therapy and short-term serious events in older adults with dementia. Arch Intern Med 2008; 168: 1090-6.

13 Kales HC, Valenstein M, Kim HM, McCarthy JF, Ganoczy D, Cunningham F, et al. Mortality risk in patients with dementia treated with antipsychotics versus other psychiatric medications. Am J Psychiatry 2007; 164: 1568-76.

14 US Food and Drug Administration. Information on Conventional Antipsychotics. US Department of Health and Human Services (http:// www.fda.gov/Drugs/DrugSafety/PostmarketDrugSafetylnformationfor PatientsandProviders/DrugSafetyInformationforHealthcareProfessionals/ PublicHealthAdvisories/ucm107211).

15 US Food and Drug Administration. Public Health Advisory: Deaths with Antipsychotics in Elderly Patients with Behavioral Disturbances. US Department of Health and Human Services (http://www.fda.gov/Drugs/ DrugSafety/PostmarketDrugSafetyInformationforPatientsandProviders/ DrugSafetyInformationforHeathcareProfessionals/PublicHealthAdvisories/ ucm053171).

16 Dorsey ER, Rabbani A, Gallagher SA, Conti RM, Alexander GC. Impact of FDA black box advisory on antipsychotic medication use. Arch Intern Med 2010; 170: 96-103.

17 Gerhard T, Lucas J, Olfson M, Hoover D, Kalay E, Crystal S. Effect of regulatory warnings on antipsychotic prescribing for nursing home residents with dementia. Pharmacoepidemiol Drug Saf 2008; 17: S120.

18 Kales HC, Zivin K, Kim HM, Valenstein M, Chiang C, Ignacio RV, et al. Trends in antipsychotic use in dementia 1999-2007. Arch Gen Psychiatry 2011; 68: 190-7.

19 Medicines and Healthcare Products Regulatory Agency. Drug Safety Update March 2009: vi. MHRA, 2009.

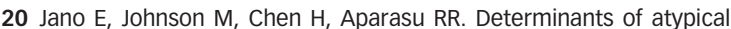
antipsychotic use among antipsychotic users in community-dwelling elderly, 1996-2004. Curr Med Res Opin 2008; 24: 709-16. 
21 Kamble P, Sherer J, Chen H, Aparasu R. Off-label use of second-generation antipsychotic agents among elderly nursing home residents. Psychiatr Serv 2010; 61: 130-6.

22 Child A, Clarke A, Fox C, Maidment I. A pharmacy led program to review anti-psychotic prescribing for people with dementia. BMC Psychiatry 2012; 12: 155.

23 Huybrechts KF, Gerhard T, Crystal S, Olfson M, Avorn J, Levin R, et al. Differential risk of death in older residents in nursing homes prescribed specific antipsychotic drugs: population based cohort study. BMJ 2012; 344 e977.

24 Kales HC, Kim HM, Zivin K, Valenstein M, Seyfried LS, Chiang C, et al. Risk of mortality among individual antipsychotics in patients with dementia. Am J Psychiatry 2011; 169: 71-9.

25 Rossom RC, Rector TS, Lederle FA, Dysken MW. Are all commonly prescribed antipsychotics associated with greater mortality in elderly male veterans with dementia? J Am Geriatr Soc 2010; 58: 1027-34.

26 Moride Y, Abenhaim L. Evidence of the depletion of susceptibles effect in non-experimental pharmacoepidemiologic research. J Clin Epidemiol 1994; 47: 731-7.

27 Ray WA. Evaluating medication effects outside of clinical trials: new-user designs. Am J Epidemiol 2003; 158: 915-20.

28 Woods SW. Chlorpromazine equivalent doses for the newer atypical antipsychotics. J Clin Psychiatry 2003; 64: 663-7.

29 Andreasen NC, Pressler M, Nopoulos P, Miller D, Ho BC. Antipsychotic dose equivalents and dose-years: a standardized method for comparing exposure to different drugs. Biol Psychiatry 2009; 67: 255-62.

30 Doody MM, Hayes HM, Bilgrad R. Comparability of national death index plus and standard procedures for determining causes of death in epidemiologic studies. Ann Epidemiol 2001; 11: 46-50.

31 Fillenbaum GG, Burchett BM, Blazer DG. Identifying a national death index match. Am J Epidemiol 2009; 170: 515-8.

32 World Health Organization. International Statistical Classification of Diseases and Related Health Problems (ICD-10). WHO, 1992.

33 Sesso HD, Gaziano JM, Glynn RJ, Buring JE. Value of an Endpoints Committee versus the use of nosologists for validating cause of death. Contemp Clin Trials 2006; 27: 333-9.

34 Schneeweiss S, Rassen JA, Glynn RJ, Avorn J, Mogun H, Brookhart MA High-dimensional propensity score adjustment in studies of treatment effects using health care claims data. Epidemiology 2009; 20: 512-22.

35 Preskorn $\mathrm{SH}$. Clinical relevance of relative receptor binding affinity: quetiapine and ziprasidone as examples. J Psychiatr Pract 2007; 13: 393-8.

36 Shayegan DK, Stahl SM. Atypical antipsychotics: matching receptor profile to individual patient's clinical profile. CNS Spectr 2004; 9 (suppl 11): 6-14.

37 Curran S, Turner D, Musa S, Wattis J. Psychotropic drug use in older people with mental illness with particular reference to antipsychotics: a systematic study of tolerability and use in different diagnostic groups. Int J Geriatr Psychiatry 2005; 20: 842-7.

38 Huybrechts KF, Brookhart MA, Rothman KJ, Silliman RA, Gerhard T, Crystal S, et al. Comparison of different approaches to confounding adjustment in a study on the association of antipsychotic medication with mortality in older nursing home patients. Am J Epidemiol 2011; 174: 1089-99.

39 Fillit H, Geldmacher DS, Welter RT, Maslow K, Fraser M. Optimizing coding and reimbursement to improve management of Alzheimer's disease and related dementias. J Am Geriatr SoC 2002; 50: 1871-8.

40 Lin PJ, Kaufer DI, Maciejewski ML, Ganguly R, Paul JE, Biddle AK. An examination of Alzheimer's disease case definitions using Medicare claims and survey data. Alzheimers Dement 2010; 6: 334-41.

41 Bradford A, Kunik ME, Schulz P, Williams SP, Singh $\mathrm{H}$. Missed and delayed diagnosis of dementia in primary care: prevalence and contributing factors. Alzheimer Dis Assoc Disord 2009; 23: 306-14.

42 Newcomer R, Clay T, Luxenberg JS, Miller RH. Misclassification and selection bias when identifying Alzheimer's disease solely from Medicare claims records. J Am Geriatr SoC 1999; 47: 215-9.

43 Corbett A, Ballard C. Antipsychotics and mortality in dementia. Am J Psychiatry 2012; 169: 7-9.

44 McCleery J, Fox R. Antipsychotic prescribing in nursing homes. BMJ 2012; 344: e1093.

45 Steinberg M, Lyketsos CG. Atypical antipsychotic use in patients with dementia: managing safety concerns. Am J Psychiatry 2012; 169: 900-6.

46 National Institute for Health and Clinical Excellence/Social Care Institute for Excellence. Dementia - Supporting People with Dementia and Their Carers in Health and Social Care. Clinical Guidance CG42. NICE/SCIE, 2006.

47 Ayalon L, Gum AM, Feliciano L, Arean PA. Effectiveness of nonpharmacological interventions for the management of neuropsychiatric symptoms in patients with dementia: a systematic review. Arch Intern Med 2006; 166: 2182-8.

48 Cohen-Mansfield J, Thein K, Marx MS, Dakheel-Ali M. What are the barriers to performing nonpharmacological interventions for behavioral symptoms in the nursing home? J Am Med Dir Assoc 2012; 13: 400-5.

49 Ballard CG, Gauthier S, Cummings JL, Brodaty H, Grossberg GT, Robert P, et al. Management of agitation and aggression associated with Alzheimer disease. Nat Rev Neurol 2009; 5: 245-55.

50 Mitka M. CMS seeks to reduce antipsychotic use in nursing home residents with dementia. JAMA 2012; 308: 119-21.

51 Banerjee S. The Use of Antipsychotic Medication for People With Dementia: Time for Action. A Report for the Minister of State for Care Services. Department of Health, 2009.

52 Pollock BG, Mulsant BH. Between Scylla and Charybdis: antipsychotic and other psychotropic medications in older nursing home residents. CMAJ 2011; 183: $778-9$. 